\title{
Internal supersymmetry and unification
}

(Lie superalgebras/fundamental representations/weak-electromagnetic charges/color/quark and lepton assignments)

\author{
YUVAL NE'EMAN ${ }^{\dagger \ddagger}$ AND SHLOMO STERNBERG ${ }^{\dagger \S}$ \\ †Tel Aviv University, Tel Aviv, Israel; ¥University of Texas, Austin, Texas 78712; and \$Harvard University, Cambridge, Massachusetts 02138
}

Contributed by Yuval Ne'eman, March 25, 1980

\begin{abstract}
We construct a family of finite-dimensional representations of the superalgebra $s(n / m)$ that depend on an integer parameter for $m>1$ and on a complex parameter, $b$, for $m=1$. We describe some models of elementary particles for $s k(2 / 1), s k(3 / 1)$, and $s k(5 / 1)$. This involves the choice of the parameter $b$ and the choice of the operators $I_{3}$ (the third component of the weak left-handed isospin) and $U$ (the weak hypercharge). These must commute, and are related to the electric charge by the usual formula $Q=I_{3}+1 / 2 U$. In particular, taking $I_{3}$ to be in its standard form in $s u(2) \subset s k(5) \subset s k(5 / 1)$ and requiring that $U$ commute with color $\operatorname{su}(3) \subset s k(5) \subset s h(5 / 1)$ leaves three free parameters, two for the choice of $U$ and one for the choice of $b$. We show that there are just two possible choices of these parameters yielding exactly all 32 quark and lepton charges: the Georgi-Glashow $U \in$ su(5), corresponding to $U(1,-2 / 3)$ and arbitrary $b$ and $U(0,1 / 3) \notin s u(5)$, with $b=2$. We provide a general construction of representations of $s k(n / 1)$ consisting exactly of sequences of generations of quarks and leptons.
\end{abstract}

1. The finite-dimensional irreducible representations of the superalgebra $s l(2 / 1)$ have been classified by Scheunert $e t$ al. (1). Among these representations there is a fundamental family of four-dimensional representations depending on a complex parameter, $\boldsymbol{b}$. We shall construct a corresponding family of representations of $s l(n / 1)$, also depending on a complex parameter, on a space of dimension $2^{n}$. Our construction will also yield a representation of $s l(n / m)$ for general values of $m$ (on a space whose dimension is somewhat more difficult to describe), provided that $b$ is a nonnegative integer. In terms of the general description of the irreducible representations of superalgebras given by Kac (2), our representations are "atypical" in the sense that their dimension is smaller than the dimension of a "typical" representation.

We begin by recalling the definition of the superalgebras $s l(n / m)$. (We refer the reader to Corwin et al. (3) for the basic general facts about superalgebras and their representations.) Let $V$ and $X$ be complex vector spaces with $\operatorname{dim} V=n$ and $\operatorname{dim}$ $X=m$. Let $W$ be the super (or graded) vector space

$$
W=V+X,
$$

in which $V$ and $X$ are given opposite parity. The superalgebra $s l(n / m)$, or $s l(V / X)$, is the algebra of all endomorphisms of $W$ of supertrace zero. A typical such endomorphism can be written as a matrix of maps

$$
\left(\begin{array}{l}
A B \\
C D
\end{array}\right), \operatorname{tr} A=\operatorname{tr} D
$$

in which $A \in \operatorname{Hom}(V, V), B \in \operatorname{Hom}(X, V), C \in \operatorname{Hom}(V, X)$, and $D \in \operatorname{Hom}(X, X)$. Those endomorphisms with $B=C=0$ are even, and those with $A=D=0$ are odd. In what follows, it will be convenient to use the identification of $\operatorname{Hom}(X, V)$ with

The publication costs of this article were defrayed in part by page charge payment. This article must therefore be hereby marked "advertisement" in accordance with 18 U. S. C. $\$ 1734$ solely to indicate this fact.
$V \otimes X^{*}$. In particular, $\nu \otimes \xi$ for $\nu \in V$ and $\xi \in X^{*}$ corresponds to the rank-one linear transformation given by

$$
(\nu \otimes \xi) \omega=\langle\xi, \omega\rangle \nu .
$$

these span $\operatorname{Hom}(X, V)$. Similarly, we identify $\operatorname{Hom}(V, X)$ with $X \otimes V^{*}$, etc. When applied to rank-one elements, we obtain the commutator

$$
\begin{aligned}
{\left[\left(\begin{array}{cc}
0 & \nu \otimes \xi \\
0 & 0
\end{array}\right),\left(\begin{array}{ll}
0 & 0 \\
x \otimes u^{*} & 0
\end{array}\right)\right] } & \\
& =\left(\begin{array}{cc}
\langle\xi, x\rangle \nu \otimes u^{*} & 0 \\
0 & \left\langle u^{*}, \nu\right\rangle x \otimes \xi
\end{array}\right) .
\end{aligned}
$$

Let $S(X)$ denote the ring of polynomial functions on $X^{*}$, so that

$$
S(X)=\overbrace{0}^{\infty} S^{k}(X)
$$

in which $S^{k}(X)$ consists of homogeneous polynomials of degree $k$. Each $x \in X$ defines a multiplication operator $m_{x}$ on $S(X)$, in which

$$
\left(m_{x} f\right)(\eta)=\langle\eta, x\rangle f(\eta), \forall \eta \in X^{*}
$$

Also, each $\xi \in X^{*}$ defines a derivation $D_{\xi}$ (differentiation in the direction $\xi$ ) determined by

$$
\begin{gathered}
D_{\xi}(f g)=\left(D_{\xi} f\right) g+f D_{\xi} g, \\
D_{\xi} l=0,
\end{gathered}
$$

and

$$
D_{\xi} x=\langle\xi, x\rangle \text { for } x \in X=S^{1}(X) .
$$

The standard commutation relations

$$
D_{\xi} m_{x}-m_{x} D_{\xi}=i d
$$

hold. Equally well, we could let $F^{b}$ denote the space of smooth functions defined on some cone in $X$ and homogeneous of degree $b$. Then $D_{\xi}: F^{b} \rightarrow F^{b-1}$ and $m_{x} F^{b} \rightarrow F^{b+1}$ and the above commutation relations hold. In particular, if $\operatorname{dim} X=1$, we can let $F^{b}$ consist of all multiples of (the formal symbol) $x^{b}$ and define $m_{x} x^{b}=x^{b+1}$ and $D_{\xi} x^{b}=b x^{b-1}$. Again the commutation relations 1.6 hold.

Let $\Lambda(V)=\underset{k=1}{\operatorname{dim} V} \Lambda^{k}(V)$ be the exterior algebra of $V$. Each $\nu \in V$ defines an operation of exterior multiplication, $e(v)$ : $\Lambda^{k}(V) \rightarrow \Lambda^{k+1}(V)$ by

$$
e(\nu) \omega=\nu \Lambda \omega .
$$

Also, each $u^{*} \in V^{*}$ defines a (super) derivation of $\Lambda(V)$, which we denote by $i\left(u^{*}\right)$. Thus $i\left(u^{*}\right): \Lambda^{k}(V) \rightarrow \Lambda^{k-1}(V)$ and is determined by

$$
i\left(u^{*}\right)\left(\omega_{1} \Lambda \omega_{2}\right)=i\left(u^{*}\right) \omega_{1} \Lambda \omega_{2}+(-1)^{\operatorname{deg} \omega_{1}} \omega_{1} \Lambda i\left(u^{*}\right) \omega_{2}
$$




$$
\begin{gathered}
i\left(u^{*}\right) \nu=\left\langle u^{*}, \nu\right\rangle, \quad \nu \in V=\Lambda^{1}(V) \\
i\left(u^{*}\right) c=0, \quad c \in C=\Lambda^{0}(V) .
\end{gathered}
$$

It is easy to check that

$$
\begin{aligned}
& e\left(\nu_{1}\right) e\left(\nu_{2}\right)+e\left(\nu_{2}\right) e\left(\nu_{1}\right)=0 \\
& i\left(u_{1}^{*}\right) i\left(u_{2}^{*}\right)+i\left(u_{2}^{*}\right) i\left(u_{1}^{*}\right)=0
\end{aligned}
$$

and

$$
e(\nu) i\left(u^{*}\right)+i\left(u^{*}\right) e(\nu)=\left\langle u^{*}, \nu\right\rangle i d .
$$

In short, $m_{x}$ and $D_{\xi}$ are Bose-Einstein creation and annihilation operators, and $e(\nu)$ and $i\left(u^{*}\right)$ are Fermi-Dirac creation and annihilation operators.

Each $A \in \operatorname{Hom}(V, V)$ determines a derivation of $\Lambda(V)$ that we continue to denote by $A$. Thus $A: \Lambda^{k}(V) \rightarrow \Lambda^{k}(V)$ is determined by

$$
\begin{aligned}
& A\left(\omega_{1} \wedge \omega_{2}\right)=A \omega_{1} \wedge \omega_{2}+\omega_{1} \wedge A \omega_{2} \\
& A c=0 \text { for } c \in \Lambda^{0}(V)=C
\end{aligned}
$$

and $A \nu$ is given as $A \nu$ on $\Lambda^{1}(V)=V$. Similarly, each $D \epsilon$ $\operatorname{Hom}(X, X)$ determines a derivation of $S(X)$. For the case dim $X=1$, in which $D$ is simply a scalar, $D$ acts on $F^{b}$ by $D\left(x^{b}\right)=$ $(b D) x^{b}$.

We now define a representation of $s l(V / X)$ on $\Lambda(V) \otimes S(X)$ by setting (see 1.1)

$$
\rho\left\{\left(\begin{array}{cc}
A & 0 \\
0 & D
\end{array}\right)\right\}=A \otimes I_{X}+I_{V} \otimes D,
$$

in which $A$ denotes the induced derivation on $\Lambda(V)$ and $I_{X}$ is the identity map on $S(X)$, with similar notations for $D$ and $I_{V}$. We set, following 1.3,

$$
\begin{gathered}
\rho\left\{\left(\begin{array}{ll}
0 & \nu \otimes \xi \\
0 & 0
\end{array}\right)\right\}=e(\nu) \otimes D_{\xi} \\
\rho\left\{\left(\begin{array}{ll}
0 & 0 \\
x \otimes u^{*} & 0
\end{array}\right)\right\}=i\left(u^{*}\right) \otimes m_{x} .
\end{gathered}
$$

Because $e\left(\nu_{1}\right) e\left(\nu_{2}\right)=-e\left(\nu_{2}\right) e\left(\nu_{1}\right)$ and $D_{\xi_{1}} D_{\xi_{2}}=D_{\xi_{2}} D_{\xi_{1}}$, it follows that

$$
\begin{aligned}
\rho\left\{\left(\begin{array}{ll}
0 & B_{1} \\
0 & 0
\end{array}\right)\right\} \cdot \rho\left\{\left(\begin{array}{ll}
0 & B_{2} \\
0 & 0
\end{array}\right)\right\} \\
+\rho\left\{\left(\begin{array}{ll}
0 & B_{2} \\
0 & 0
\end{array}\right)\right\} \cdot \rho\left\{\left(\begin{array}{ll}
0 & B_{1} \\
0 & 0
\end{array}\right)\right\}=0
\end{aligned}
$$

and similarly

$$
\begin{array}{r}
\rho\left\{\left(\begin{array}{ll}
0 & 0 \\
C_{1} & 0
\end{array}\right)\right\} \cdot \rho\left\{\left(\begin{array}{ll}
0 & 0 \\
C_{2} & 0
\end{array}\right)\right\} \\
+\rho\left\{\left(\begin{array}{ll}
0 & 0 \\
C_{2} & 0
\end{array}\right)\right\} \cdot \rho\left\{\left(\begin{array}{ll}
0 & 0 \\
C_{1} & 0
\end{array}\right)\right\}
\end{array}
$$

Also,

$$
\left(e(\nu) \otimes D_{\xi}\right) \cdot\left(i\left(u^{*}\right) \otimes m_{x}\right)=\left(\nu \otimes u^{*}\right) \otimes\left(\langle\xi, x\rangle I_{X}+x \otimes \xi\right)
$$

and

$$
\left(i\left(u^{*}\right) \otimes m_{x}\right) \cdot\left(e(\nu) \otimes D_{\xi}\right)=\left(\left\langle u^{*}, \nu\right\rangle I_{V}-\nu \otimes u^{*}\right) \otimes(x \otimes \xi),
$$

which proves that

$$
\begin{aligned}
\rho\left\{\left(\begin{array}{ll}
0 & B_{1} \\
C_{1} & 0
\end{array}\right)\right\} & \rho\left\{\left(\begin{array}{ll}
0 & B_{2} \\
C_{2} & 0
\end{array}\right)\right\} \\
+ & \rho\left\{\left(\begin{array}{ll}
0 & B_{2} \\
C_{2} & 0
\end{array}\right)\right\} \cdot \rho\left\{\left(\begin{array}{ll}
0 & B_{1} \\
C_{1} & 0
\end{array}\right)\right\} \\
& =\rho\left\{\left[\left(\begin{array}{cc}
0 & B_{1} \\
C_{1} & 0
\end{array}\right),\left(\begin{array}{ll}
0 & B_{2} \\
C_{2} & 0
\end{array}\right)\right]\right\}
\end{aligned}
$$

The remaining bracket relations are straightforward to verify.

In particular, for each integer $k$, the space

$$
\begin{aligned}
& \Lambda^{0}(V) \otimes S^{k}(X)+\Lambda^{1}(V) \otimes S^{k-1}(X) \\
&+\ldots+\Lambda^{n}(V) \otimes S^{k-n}(X)
\end{aligned}
$$

gives a finite dimensional irreducible representation of $s l(V / X)$ where $n=\operatorname{dim} V$. Here $S^{j}(X)$ is taken to be $\{0\}$ if $j<0$. [Thus, for $k=1$ we obtain the basic defining representation of $\operatorname{sl}(\mathrm{V} / \mathrm{X})$.]

In the case $\operatorname{dim} X=1$, we can, for each $b \in C$, consider the representation on the space

$$
\Lambda^{0}(V) \otimes F^{b}+\ldots+\Lambda^{n}(V) \otimes F^{b-n},
$$

which has the same dimension $2^{n}$ as $\Lambda(V)$. For the case $\operatorname{dim} V$ $=2$, these are the representations of Scheunert et al. (1) mentioned above. (For $\operatorname{dim} X>1$ these spaces will be infinite dimensional.)

2. In refs. 4 and 5 , the superalgebra $s l(2 / 1)$ was used as an internal supersymmetry of the standard-model unified weakelectromagnetic gauge. That is, $\operatorname{dim} V=2$, and $\operatorname{dim} X=1$. The operators $U$ and $I_{3}$ were chosen as

$$
\begin{gathered}
U=\left(\begin{array}{cc|c}
1 & 0 & 0 \\
0 & 1 & 0 \\
\hline 0 & 0 & 2
\end{array}\right) \\
I_{3}=\left(\begin{array}{cc|c}
1 / 2 & 0 & 0 \\
0 & -1 / 2 & 0 \\
\hline 0 & 0 & 0
\end{array}\right)
\end{gathered}
$$

and the electric charge operator was taken to be $I_{3}+1 / 2 U$. For reasons of space, we shall write these matrices and similar ones in what follows as

$$
U=\operatorname{diag}(1,1, \mid 2) \text { and } I_{3}=\operatorname{diag}(1 / 2,-1 / 2 \mid 0)
$$

The value $b=2 / 3$ then corresponded to the (Cabibbo-rotated) nonstrange quarks with eigenvalues and particle assignments (up to statistics, see Discussion)

$$
\begin{aligned}
& U^{\frac{\Lambda^{0}}{4 / 3}} \frac{\Lambda^{1}}{1 / 3} \frac{\Lambda^{2}}{-2 / 3} \\
& \begin{array}{lllll}
I_{3} & 0 & 1 / 2 & -1 / 2 & 0
\end{array} \\
& \begin{array}{llll}
u_{R}^{2 / 3} & u_{L}^{2 / 3} & d_{L}^{-1 / 3} & d_{R}^{-1 / 3}
\end{array}
\end{aligned}
$$

The value $b=1$ corresponds to the anti-lepton assignments

$$
\begin{array}{ccccc}
U & \frac{\Lambda^{0}}{2} & \multicolumn{2}{c}{\Lambda^{1}} & \frac{\Lambda^{2}}{0} \\
I_{3} & 0 & 1 & 1 & 0 \\
& \left(e_{R}\right)_{L} & \left(e_{L}\right)_{R} & -1 / 2 & 0 \\
& \left(\nu_{L}^{0}\right)_{R} & \left(\nu_{R}^{0}\right)_{L}
\end{array}
$$

The choices $b=1 / 3$ and $b=0$ correspond respectively to the charge-conjugate multiplets (antiquarks and leptons). The representations $b=1,0$ are reducible, with $\Lambda^{0}+\Lambda^{1}$ and $\Lambda^{0}$ respectively an invariant subspace. We shall discuss this point later. The same sort of representations $b=2 / 3,1,1 / 3,0$ will describe any other "generation" of quarks and leptons.

3. We now point out that we can combine the quarks and leptons into a single, eight-dimensional representation of $s l(3 / 1)$. Indeed, choose

$$
\begin{aligned}
& U=\operatorname{diag}(1 / 3,1 / 3,2 / 3 \mid 4 / 3) \\
& I_{3}=\operatorname{diag}(1 / 2,-1 / 2,0 \mid 0)
\end{aligned}
$$


For $b=3 / 2$ we get the eigenvalues

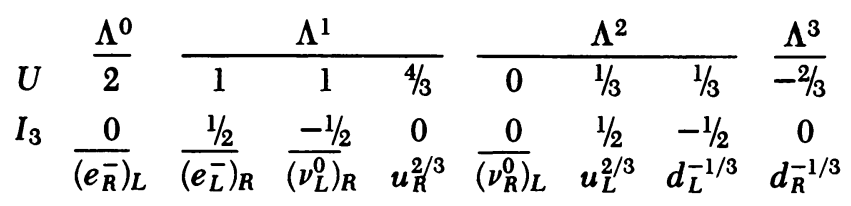

and the representation restricts under $s l(2 / 1)$ as indicated.

For $b=1 / 2$ we get the conjugate eigenvalues

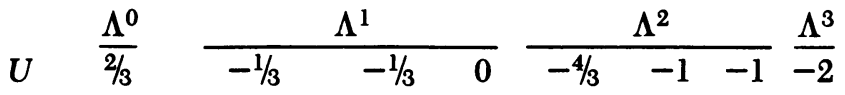

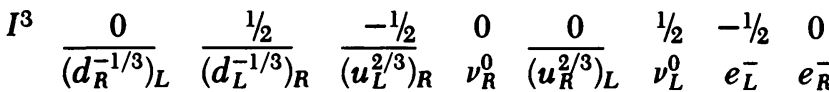

We observe that, because $b$ is not an integer, these representations are irreducible.

Turning now to a unification of asthenodynamic (weakelectromagnetic) charges with color $S U(3)$, we choose in $s l(5 / 1)$ the generators

$$
U=\operatorname{diag}(0,0,1 / 3,1 / 3,1 / 3 \mid 1)
$$

and

$$
I_{3}=\operatorname{diag}(1 / 2,-1 / 2,0,0,0 \mid 0)
$$

and the representation $b=2$. This is a 32-dimensional (reducible-see Dicussion) representation with the assignments given in 3.7 in the Appendix (the lower index on the left of a quark denotes color) with the additional quantum number $N$ corresponding to the generator

$$
N=\operatorname{diag}(1,1,-2 / 3,-2 / 3,-2 / 3 \mid 0) \text {. }
$$

To deal with more than one generation we turn to $s l(6 / 1)$, $b=5 / 2$. This representation is irreducible because $b$ is not an integer. We choose the generators

$$
\begin{aligned}
& U=\operatorname{diag}\left(-1 / 5,-1 / 5,4 / 5,2 / 15,2 / 15,2 /\left.15\right|^{4 / 5}\right) \\
& I_{3}=\operatorname{diag}(1 / 2,-1 / 2,0,0,0,0 \mid 0) \\
& N=\operatorname{diag}(1,1,1,-1,-1,-1 \mid 0) \\
& Y=\operatorname{diag}(1,1,-2,0,0,0 \mid 0) .
\end{aligned}
$$

The associated eigenvalues are given in 3.10 in the Appendix (the lower index ${ }_{3}$ denotes color multiplicity),

In $s l(7 / 1)$ we would choose

$$
U=\operatorname{diag}(-1 / 3,-1 / 3,2 / 3,2 / 3,0,0,0 \mid 2 / 3), b=3 \text {. }
$$

The same eigenvalues as for $s l(6 / 1)$ occur, but with double the multiplicity. Thus each 2 occurs four times (once in $\Lambda^{0}$, twice in $\Lambda^{1}$, and once in $\Lambda^{2}$ ), etc., corresponding to four generations of quarks and leptons.

4. Discussions. The maximal even Lie subalgebra of $s l(5 / 1)$ is $s l(5) \times C$ or $s u(5) \times u(1)$ when reducing to the unitary subalgebra. Aside from $U, I_{3}$, and the two quantum numbers of the Cartan subalgebra of $s u(3)$, we dispose of a fifth generator

" An earlier attempt to unify $s u(2 / 1)$ with color by D. Fairlie, Y. Ne'eman, and $\mathrm{Dj}$. Sijački in May 1979 failed to provide a general proof of the existence of such representations and their construction. However, these authors proved for $s u(5 / 1)$ and other candidates that no representations exist with only leptons and quarks (and no antiparticles). The 16-dimensional $\Lambda$ representation with $U \rightarrow N$ of 3.8 was noted and abandoned when it proved impossible to retain the unrenormalized value $\sin ^{2} \theta_{w}=1 / 4$ of $s u(2 / 1)$, in the as-yet-unproved conjecture of a conserved angle.
$N$. Note that in their $s u(5)$ unification, Georgi and Glashow (6) use $\boldsymbol{N}$ for their weak hypercharge. This provides for an alternative identification of the quarks and leptons in 3.7. These two are the only possible choices of $U$ (up to sign) that give the observed spectrum.

Notice that our $\operatorname{sl}(5 / 1)$ representation 3.7 with $b=2$ is not irreducible. The subspace $\Lambda=\Lambda^{0}+\Lambda^{1}+\Lambda^{2}$ is invariant but has no invariant complement. The same is true (e.g., 2.4) as we saw of the subspace $\Lambda^{0}+\Lambda^{1}$ in $b=1$ and of $\Lambda^{0}$ in $b=0$ of $s u(2 / 1)$ : Ne'eman's leptons $\left(\nu_{L}^{0}, e_{L}^{-}, e_{R}^{-}\right)$were assigned to such a three-dimensional invariant subspace. Indeed, the fact that the fourth state ( $\nu_{R}^{0}$ here) drops out for integer-charge multiplets (while fractional-charge ones require all four states) is in itself a remarkable "prediction" of $s u(2 / 1)$.

These peculiar reducibility phenomena are an illustration of the fact that representations of simple superalgebras need not be completely reducible. From this point of view, the particles in $\Lambda^{3}, \Lambda^{4}$, and $\Lambda^{5}$ of 3.7 seem "more fundamental," in the sense that starting from the lowest $U$ state $e_{R}^{-}$, for example, we can reach all other states, but not when starting with the highest $U$, i.e., from $\overline{\left(e_{R}^{-}\right)_{L}}$.

The above irreducible subspace $\tilde{\Lambda}$ is 16 -dimensional and if one adopts the Georgi-Glashow choice of $U \rightarrow N, \tilde{\Lambda}$ yields the representation used by Taylor (7).

As in $s u(2 / 1)$, the even-odd gradings $k$ in $\Lambda^{k}$ are correlated with the chiralities throughout our $s l(3 / 1)$ and $s l(5 / 1)$. This is nontrivial, because there is no preassigned correlation between quarks and leptons. The correlation subsists in 3.7 with either choice of $U$.

The alternating statistics corresponding to even-odd $k$ in $\Lambda^{k}$ imply "ghost" status for one-half of all states in these representations. We shall not dwell on this issue here, because our results are independent of the interpretation of these ghost states-whether as Faddeev-Popov ghosts $(4,8)$ or some other mechanism (5). It should be noted, however, that the doubling of matter fields in 3.7 and 3.10 provides for a natural realization of Ne'eman's odd (discrete) morphism $\epsilon$ used in refs. 4 and 8 to double the dimensionalities of $s u(2 / 1)$ representations.

These representations with sequential quark-lepton structure exist for any $s l(n / 1)$, with dimensionality $2^{n}$. The number of generations is $2^{4}, s=n-5$, and the quantum numbers are given by

$$
\begin{aligned}
U & =\operatorname{diag}\left(\left(\frac{-s}{4+s}, \frac{-\Delta}{4+s},\left(\frac{4}{4+s}\right)\right)_{\Delta \text { times }},\right. \\
& \left.\left.\left(\frac{4-2 s}{3(4+s)}\right)_{3 \text { times }} \mid \frac{4}{4+s}\right)\right) \\
I_{3} & =\operatorname{diag}\left(\left(1 / 2,-1 / 2,(0)_{(\Delta+3) \text { times }} \mid 0\right)\right) \\
b & =\frac{4+s}{2} .
\end{aligned}
$$

For $s l(8 / 1), s=3$ and the 8 generations are related by a (seriality) $s u(3)_{\triangleleft}$ subalgebra,

$$
\begin{aligned}
& s l(8 / 1) \supset s u(8) \times u(1), \\
& \qquad s u(8) \supset s u(2)_{L} \times s u(3)_{\downarrow} \times s u(3)_{\text {color }} .
\end{aligned}
$$

Finally, we might speculate that $V \oplus X$ constitutes the primitive field (9). For $s l(7 / 1)$ this would consist of an isodoublet $\left(\alpha_{L}^{1 / 3}, \alpha_{L}^{-2 / 3}\right)$, two seriality isosinglets $\alpha_{R, a}^{1 / 3}(a=1,2)$ and three color isosinglets $\alpha_{R, i}^{0}(i=1,2,3)$, all fermions, and a boson (ghost) $\beta_{R}^{1 / 3}$. In $s l(8 / 1)$ the charges involve multiples of $e / 21$ :

$\alpha_{L}^{2 / 7}, \quad \alpha_{L}^{-5 / 7}, \quad \alpha_{R a}^{2 / 7}(a=1 \ldots 3)$,

$$
\alpha_{R i}^{-1 / 21}(i=1 \ldots 3), \quad \text { and } \beta_{R}^{2 / 7} \text {. }
$$


Appendix.

\begin{tabular}{ccccccc}
$U$ & \multicolumn{5}{c}{$\Lambda^{0}$} & \multicolumn{5}{c}{$\Lambda^{1}$} \\
\cline { 2 - 6 }$I_{3}$ & 0 & 1 & 1 & $4 / 3$ & $4 / 3$ & $4 / 3$ \\
$N$ & 0 & & $-1 / 2$ & 0 & 0 & 0 \\
& $\frac{1}{\left(e_{R}^{-}\right)_{L}}$ & $\frac{1}{\left(e_{L}^{-}\right)_{R}}$ & $\frac{1}{\left(\nu_{L}^{0}\right)_{R}}$ & $-2 / 3$ & $-2 / 3$ & $-2 / 3$ \\
& & & $u_{R}^{2 / 3}$ & $2 u_{R}^{2 / 3}$ & ${ }_{3} u_{R}^{2 / 3}$
\end{tabular}

\begin{tabular}{|c|c|c|c|c|c|c|c|c|c|}
\hline & & & & & & & & & \\
\hline 0 & $1 / 3$ & $1 / 3$ & $1 / 3$ & $1 / 3$ & $1 / 3$ & $1 / 3$ & $2 / 3$ & $2 / 3$ & $2 / 3$ \\
\hline 0 & $1 / 2$ & $1 / 2$ & $1 / 2$ & $-1 / 2$ & $-1 / 2$ & $-1 / 2$ & 0 & 0 & 0 \\
\hline 2 & $1 / 3$ & $1 / 3$ & $1 / 3$ & $1 / 3$ & $1 / 3$ & $1 / 3$ & $-4 / 3$ & $-4 / 3$ & $-4 / 3$ \\
\hline & ${ }_{1} u_{L}^{2 / 3}$ & ${ }_{2} u_{L}^{2 / 3}$ & ${ }_{3} u_{L}^{2 / 3}$ & ${ }_{1} d_{L}^{-1 / 3}$ & ${ }_{2} d_{I}^{-1 / 3}$ & ${ }_{3} d_{I}^{-1 / 3}$ & $\overline{\left(d^{-1 / 3}\right)}$ & $\overline{\left({ }_{2} d_{B}^{-1 / 3}\right)_{L}}$ & ${\overline{\left({ }_{3} d_{R}^{-1 / 3}\right)}}_{L}$ \\
\hline
\end{tabular}

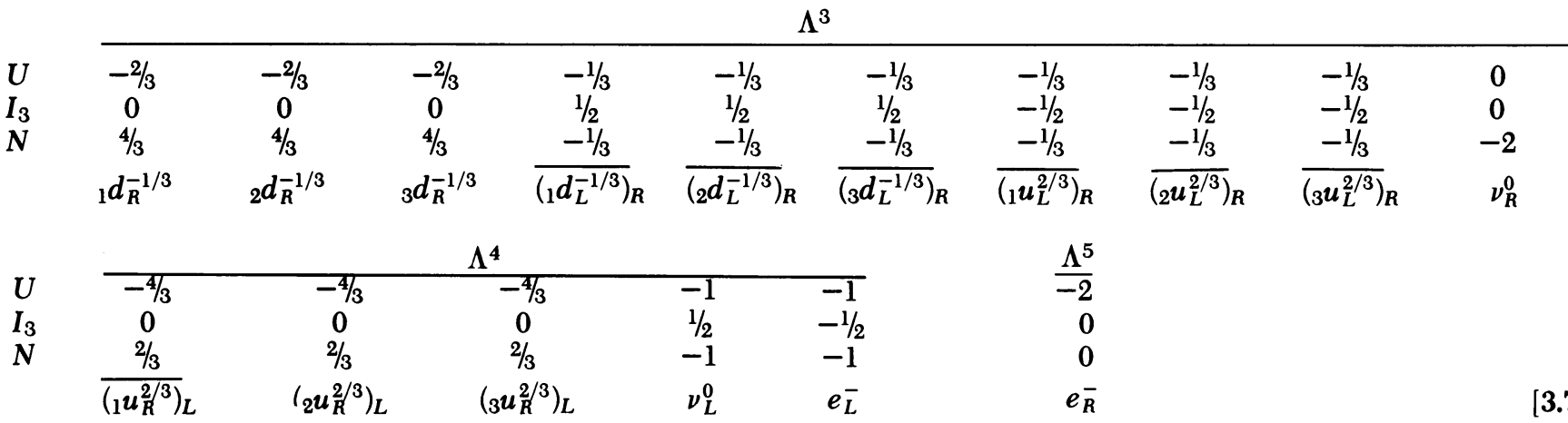

\begin{tabular}{|c|c|c|c|c|c|c|c|c|c|c|c|c|c|}
\hline$\Lambda^{0}$ & \multicolumn{6}{|c|}{$\Lambda^{1}$} & \multicolumn{7}{|c|}{$\Lambda^{2}$} \\
\hline$\overline{2}$ & $\overline{1}$ & 1 & 2 & $4 / 3$ & $4 / 3$ & $4 / 3$ & $\overline{0}$ & 1 & 1 & $(1 / 3)_{3}$ & $(1 / 3)_{3}$ & $(4 / 3)_{3}$ & $(2 / 3)$ \\
\hline$I_{3}$ & $1 / 2$ & $-1 / 2$ & 0 & 0 & 0 & 0 & 0 & $1 / 2$ & $-1 / 2$ & $(1 / 2)_{3}$ & $(-1 / 2)_{3}$ & $0_{3}$ & $\mathrm{O}_{3}$ \\
\hline 0 & 1 & 1 & 1 & -1 & -1 & -1 & 2 & 2 & 2 & $0_{3}$ & $0_{3}$ & $0_{3}$ & $-2_{3}$ \\
\hline 0 & 1 & 1 & -2 & 0 & 0 & 0 & 2 & -1 & -1 & $1_{3}$ & $l_{3}$ & $-2_{3}$ & $\mathrm{O}_{3}$ \\
\hline
\end{tabular}

$\begin{array}{lllllllll}U & 0 & (-2 / 3)_{3} & (1 / 3)_{3} & (1 / 3)_{3} & (-1 / 3)_{3} & (-1 / 3)_{3} & (2 / 3)_{3} & 0 \\ I_{3} & 0 & 0_{3} & (1 / 2)_{3} & (-1 / 2)_{3} & (1 / 2)_{3} & (-1 / 2)_{3} & 0_{3} & 0 \\ N & 3 & 1_{3} & 1_{3} & 1_{3} & -1_{3} & -1_{3} & -1_{3} & -3 \\ Y & 0 & 2_{3} & -1_{3} & -1_{3} & 1_{3} & 1_{3} & -2_{3} & 0\end{array}$

\begin{tabular}{|c|c|c|c|c|c|c|c|c|c|c|c|c|}
\hline & \multicolumn{7}{|c|}{$\Lambda^{4}$} & \multicolumn{4}{|c|}{$\Lambda^{5}$} & $\underline{\Lambda^{6}}$ \\
\hline$U$ & $(-2 / 3)_{3}$ & $(-4 / 3)_{3}$ & $(-1 / 3)_{3}$ & $(-1 / 3)_{3}$ & -1 & -1 & 0 & $(-4 / 3)_{3}$ & -2 & -1 & -1 & $\overline{-2}$ \\
\hline$I_{3}$ & $0_{3}$ & $0_{3}$ & $(1 / 2)_{3}$ & $(-1 / 2)_{3}$ & $1 / 2$ & $-1 / 2$ & 0 & $0_{3}$ & 0 & $1 / 2$ & $-1 / 2$ & 0 \\
\hline$N$ & 23 & $0_{3}$ & $0_{3}$ & $\mathrm{O}_{3}$ & -2 & -2 & -2 & $1_{3}$ & -1 & -1 & -1 & 0 \\
\hline$Y$ & $0_{3}$ & 23 & $-1_{3}$ & $-1_{3}$ & 1 & 1 & -2 & $0_{3}$ & 2 & -1 & -1 & 0 \\
\hline
\end{tabular}

Note Added in Proof. ( $i$ ) For the cases of reducible representations-i.e, where $b=(n-1) / 2$ is integral, it might be desirable to replace the reducible representation by the direct sum of the irreducible subrepresentation and the corresponding quotient representation. This would have the effect of restoring the symmetry between particles and antiparticles. (ii) The choice $b=(n-1) / 2$ can be independently justified by the requirement that there exist a nondegenerate pairing between $\Lambda^{k}$ and $\Lambda^{n-k}$. The eigenvalues of $\operatorname{diag}(1, \ldots, 1 / n)$ would have to be opposite on these two spaces, and this easily implies that $b=(n-1) / 2$. This fact was pointed out to us by $O$. Gabber. In fact, because $\Lambda^{n} \otimes F^{-1}$ has a natural trivialization under the even part of $\operatorname{sl}(n / 1)$ we see that the natural multiplication of $\Lambda^{k} \otimes F^{b-k} \times$ $\Lambda^{n-k} \otimes F^{b-(n-k)}$ into $\Lambda^{n} \otimes F^{2 b-n}$ gives a bilinear pairing when $b=(n$ $-1) / 2$. One can check that this pairing is superinvariant under all of $s l(n / 1)$. (iii) If we assume a $U$ of the form given above, and the observed charges, one can deduce that either there is no color symmetry or that the color group is $u(3)$. Details will be presented elsewhere. (iv) The Georgi-Glashow choice of $U$ can also be made for arbitrary $n \geq$
5. (v) J. Thierry-Mieg (personal communication) has informed us that a construction similar to our 1.12 has been suggested by $P$. H. Dondi and P. D. Jarvis. Apparently for $n=5$ they take $b=4$ with the $N \in$ $s u(5)$ as weak hypercharge as in ref. 7 . We have not seen their article, however. Constructions similar to 1.12 were used in "Extended Supergravity" by M. Gell-Mann and Y. Ne'eman (1976, unpublished) as quoted by Freeman (10).

This work was supported by the Wolfson Chair Extraordinary in Theoretical Physics at Tel Aviv University, by the United States-Israel Binational Science Foundation, by the United States Department of Energy (contracts DE/AS0278ER04742 and EY-76-S-05-3992), and by the Israel National Academy of Sciences and Humanities.

1. Scheunert, M., Nahm, W. \& Rittenberg, V. (1977) J. Math. Phys. 18, 155-162. 
2. Kac, V. G. (1978) in Differential Geometry Methods in Mathematical Physics II, 1977, Lecture Notes in Mathematics, No. 676 (Springer, Berlin), pp. 597-626.

3. Corwin, L., Ne'eman, Y. \& Sternberg, S. (1975) Rev. Mod. Phys. 47, 573-604.

4. Ne'eman, Y. (1979) Phys. Lett. B 81, 190-194.

5. Fairlie, D. B. (1979) Phys. Lett. B 82, 97-100.
6. Georgi, H. \& Glashow, S. L. (1974) Phys. Rev. Lett. 32, 438440.

7. Taylor, J. G. (1979) Phys. Rev. Lett. 43, 824-826.

8. Ne'eman, Y. \& Thierry-Mieg, J. (1980) Proc. Natl. Acad. Sci. USA 77, 720-723.

9. Ne'eman, Y. (1979) Phys. Lett. B 82, 69-70.

10. Freeman, D. Z. (1977) Phys. Rev. Lett. 38, 105-108. 